\title{
Normal Lymphocyte Transfer and Lymphocyte Stimulation as Practical Histocompatibility Tests*
}

\author{
G. J. JOHNSON, $\dagger$ M.B., B.CHIR. ; P. S. RUSSELL, $\dagger$ M.D.
}

Brit. med.F., 1967, 2, 202-205

This report summarizes some experience with the practical application of the normal lymphocyte transfer test (Brent and Medawar, 1963 ; Gray and Russell, 1963) and the in-vitro lymphocyte stimulation test (Bain et al., 1964 ; Bach and Hirschhorn, 1964) to the problems of selecting human donors for kidney allografts. It also compares the results obtained by the two tests in test selection from 10 panels of potential donors.

\section{Methods}

\section{Preparation of Lymphocytes for Injection}

Iron Powder Method.-The red cells were sedimented from heparinized blood either by leaving the tube to stand at $37^{\circ} \mathrm{C}$. or by the addition of gelatin. The supernatant plasma was incubated with iron powder for one hour and the iron-containing granulocytes were removed by differential sedimentation. The supernatant contained lymphocytes, some granulocytes, and the nuclei of disrupted monocytes. The washed cells were resuspended in Eagle's serum.

Nylon Filter Method.-Four hundred and fifty millilitres of blood collected in a Fenwal pack with heparin anticoagulation was centrifuged at 500 r.p.m. until the supernatant was just clear of red cells. The supernatant plasma was rewarmed to $37^{\circ} \mathrm{C}$. and passed through a Fenwal leucocyte filter containing nylon wool. This allowed lymphocytes and any residual red cells to pass. It removed $75 \%$ of the platelets. Only occasional granulocytes and rare monocytes were seen in the final preparation. It was necessary to add further heparin to the plasma and lymphocytes after their passage through the filter. When this method was used for a future graft recipient with a low haematocrit the blood taken was replaced with a unit of frozen blood (Huggins, 1964) in order to reduce to a minimum the risk of specific sensitization.

\section{Purity of Lymphocyte Preparations}

All 17 uraemic patients studied had a leucocytosis of varying degree, associated with a decreased differential lymphocyte count averaging $14 \%$. In two individuals the lymphocytes comprised only $1 \%$ of the total white cells. Furthermore, the granulocytes from these patients did not take up iron powder as readily as those from healthy controls. These factors made it difficult to achieve satisfactory lymphocyte preparations by the iron powder method. Occasional preparations were of a high degree of purity, but the average granulocyte contamination of $50.1 \%$ in the presence of uraemia was not thought acceptable for routine use.

\begin{tabular}{l|c|c|c|c}
\multicolumn{2}{c}{ TABLE I.-Purity of Lymphocyte Preparations } \\
\hline \multirow{1}{*}{ Method } & Patients & No. & Percentage of Granulocytes \\
& & & Range & Mean \\
\hline Iron powder & $\left\{\begin{array}{l}\text { Normal } \\
\text { Filter }\end{array}\right.$ & 24 & $3.0-52.0$ & 25.5 \\
Uraemic & 10 & $20.0-80.0$ & 50.1 \\
Normal & 16 & $0-7.3$ & 1.6 \\
Uraemic & 9 & $0.8-38.8$ & 13.4 \\
\hline
\end{tabular}

The nylon filter greatly improved the results (Table I) and increased the yield from ill patients. Nevertheless, it appeared that granulocytes from a uraemic patient behaved abnormally also in regard to their adherence to the surface presented by the nylon.

\section{Technique of Injection}

The lymphocytes were injected intradermally with a No. 27 needle, the injection sites being grouped fairly close together about one-third of the way down the flexor surface of the forearm from the elbow. If duplicate injections were too widely spread the reaction nearer the wrist tended to be smaller than the proximal one. Each injection delivered five million lymphocytes in $0.1 \mathrm{ml}$. of Eagle's medium. The medium alone produced no reaction.

Care was necessary to make the injections as superficial as possible, since the epidermis varied in thickness and toughness with age and sex. When the $0.1 \mathrm{ml}$. had been correctly introduced a discrete bleb, about $9 \mathrm{~mm}$. in diameter, with clear-cut edges, should result. Harris et al. (1966) have also emphasized the importance of an exact technique for the injection.

\section{Measurement of Skin Reactions}

The maximum and minimum diameters of the indurated areas at 48 hours were used for most of the studies. In the later tests accurate measurement of the surface area of the induration in square millimetres was also made by the method of Moorhead and Patel (1964).

\section{Use of Controls in the Test}

If aliquots of a single lymphocyte suspension are injected in identical fashion into the skin of the forearms of several recipients, unrelated to the lymphocyte donor, lesions of widely differing size are usually produced. These differences reflect not only differences in histocompatibility but also a variety of independent physiological variables in the reactivity of the recipients' skins. The absolute sizes of the reactions produced in each cell recipient by the lymphocytes being tested are therefore of limited value. Controls must be introduced to enable them to be interpreted.

The design of a standard normal lymphocyte transfer test in this laboratory is represented in the Diagram. Two, or preferably three, control lymphocyte suspensions from healthy individuals unrelated to the lymphocyte recipients or to the patient were injected near the injection sites for the patients' lymphocytes. At least one of these individuals could be expected to be quite different from each lymphocyte recipient with respect to histocompatibility antigens, and the control injections could therefore be expected to elicit at least one example

* This work was supported in part by grants AM-07055 and A1-06320 from the United States Public Health Service.

t Department of Surgery, Harvard Medical School, and the General Surgical Services of the Massachusetts General Hospital 
of the maximal physiological response for each recipient. In practice these control inocula usually produced closely similar reactions in a particular forearm.

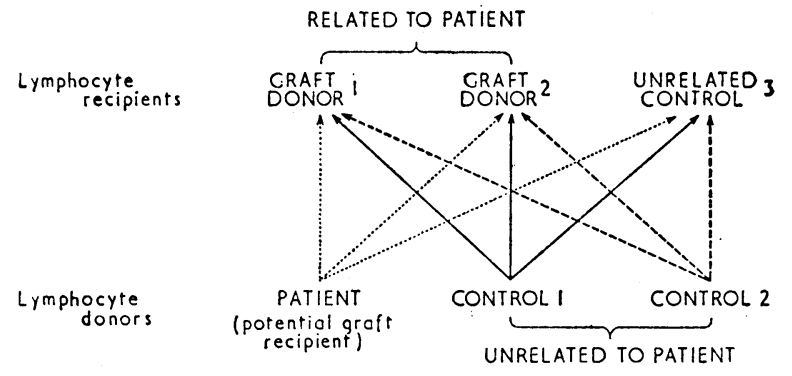

Plan of normal lymphocyte transfer test.

The lesion produced by the patient's cells could then be compared with this maximum "baseline" in each case. For example, if a possible donor produced as big a reaction to the patient's cells as to the control injections he was thought to be a poor match.

At least one unrelated control individual (Control 3 in Diagram) was also included among the lymphocyte recipients. Unless he happened to be a chance good match the patient's cells should produce in his arm a somewhat smaller reaction than the controls if they were less reactive owing to uraemia or some other nonspecific factor. This control could similarly reveal nonspecific hyperreactivity of the patient's lymphocyte preparation.

\section{Method of Lymphocyte Culture}

The technique was modified from Hirschhorn (1965). The cultured lymphocytes were prepared by the iron powder method; by leaving the heparinized blood to stand at $37^{\circ} \mathrm{C}$. for over 40 minutes so that many granulocytes sedimented with the red cells ; or by passing the plasma containing mixed white cells through a smaller-scale version of the filter, a cotton plug in a glass tube (Streilein, 1966). Lymphocytes passed through the nylon filter could usually not be made to undergo mitosis in culture.

When the cells were grown in Hirschhorn's original medium a high degree of " spontaneous" lymphoblastic change occurred in the control tubes. In later experiments the antibiotics were removed, and the foetal calf serum was replaced with autologous human serum. The control counts were then regularly reduced to a very low level (Johnson and Russell, 1965).

The tubes of mixed lymphocytes contained equal quantities of living donor and recipient ceils. Controls and mixtures were put up in quadruplicate and the average reading was used. The results were expressed as the net percentage of large cells plus mitoses-that is, the precentage in the mixed cultures minus the average of those in the corresponding controls.

An alternative methed of measuring results consisted in exposing each tube to $4 \mathrm{ml}$. of tritiated thymidine at the end of the seven-day culture period. Radioactivity of the washed cells was counted in a scintillation counter (modified from Chen, 1958).

\section{Preliminary Findings}

\section{Reproducibility of Normal Lymphocyte Transfer Injections}

The extent of the induration produced by the injections was taken as the product of the maximum and minimum diameters (in millimetres) and expressed as an index. The standard deviation for duplicate and triplicate injections from 29 different preparations-including some from uraemic patients-was 3.7 .
Influence of Method of Preparation.-For this analysis preparations of cells from 40 normal non-uraemic persons-for example, Controls 1 and 2 in the Diagram-were included, and only the reactions which these cells produced in unrelated normal persons were considered. The two groups of reactions being compared were therefore produced under closely similar conditions, except that the cells were prepared by either the iron powder or the nylon filter method. The mean indices of induration of 119 reactions are compared in Table II. There was no significant difference between the two methods. The nylon filter can therefore be said to provide a satisfactory method of separating lymphocytes from ill patients with low lymphocyte counts which does not impair their reactivity.

\begin{tabular}{|c|c|c|c|}
\hline \multicolumn{2}{|c|}{ Method of Preparation } & \multirow{2}{*}{ 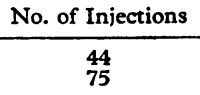 } & \multirow{2}{*}{ 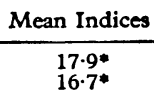 } \\
\hline $\begin{array}{l}\text { Iron powder } \\
\text { Nylon filter }\end{array}$ & $\begin{array}{ll}. . & \cdots\end{array}$ & & \\
\hline
\end{tabular}

Importance of Granulocyte Contamination.-The same 119 measurements were used as above. Two curves were drawn, one for each method of lymphocyte preparation, the size of each reaction being plotted against the percentage of granulocytes in the corresponding lymphocyte suspension. There was no correlation between the two variables. This supports the finding of Bridges et al. (1964) that the granulocyte contamination does not influence the size of the reaction.

Effect of Uraemia.-Bridges et al. (1964) also noted that cells from uraemis individuals were more likely to produce a reaction under $5 \mathrm{~mm}$. of induration than cells from normal people. To assess the influence of uraemia, 19 normal subjectsfor example, Control 3 in the Diagram-were selected who had received simultaneous injections from two or three unrelated normal individuals and from unrelated uraemic patients (blood urea nitrogen greater than $70 \mathrm{mg} . / 100 \mathrm{ml}$. ; mean $176 \mathrm{mg} . / 100$ $\mathrm{ml}$.). The size of the lesions produced by the uraemic cells was plotted against the mean for the reactions produced by normal cells in the same individual. No systematic relation was revealed. This suggests that uraemia per se did not regularly diminish the size of the normal lymphocyte transfer reaction.

Preliminary Studies with In-vitro Lymphocyte Stimulation. -When the mixed lymphocyte cultures for a given panel were repeated a number of times the absolute uptake of tritiated thymidine for the different pairs of cells varied considerably. However, the order of reactivity, and therefore the order of donor preference, remained constant from one experiment to the next (Russell, 1966). In five small panels of volunteers the mean survival times of skin grafts exchanged between members of the panel were predicted beforehand on the basis of the results of the mixed lymphocyte culture. This prediction was correct in every instance except one, where a difference of half a day in mean skin-graft survival time between two pairs of exchanges was not forecast by the cultures.

\section{Results}

Ten panels, of varying size, of potential kidney donors were studied by both tests. For each test the donors were arranged in order of desirability in regard to compatibility. The two banks of relative compatibility order for each panel were then compared; the results are given in Table III.

Both tests choose the same donor as the most compatible in the first eight panels, and in all but one the second choice coincides also. On the right side of Table III, however, the two tests do not in general agree in allocating " degrees of undesirability." The data were therefore rearranged so that the tests were required to agree only in distinguishing "good" from "fairly good" and "poor" donors (according to the 
definitions of Table IV). The correspondence between the two tests then became closer. Of 33 donors there were only five in which discrepancies existed between the matching by the two methods when expressed in these terms.

TABLE III.-Correspondence of Order of Ranking of Donors by Normal Lymphocyte Transfer and Lymphocyte Stimulation Tests

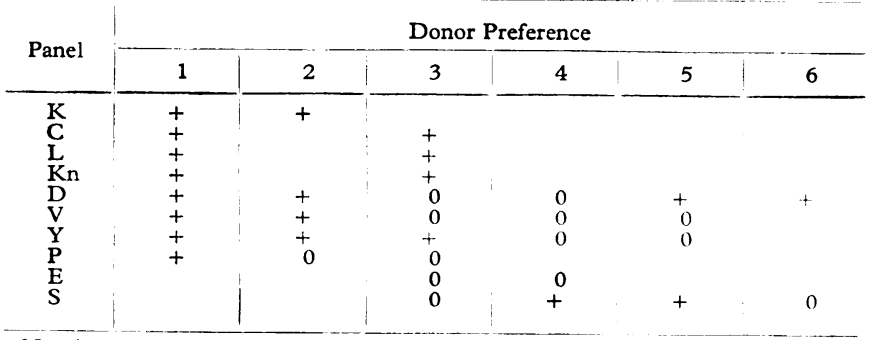

Number of symbols $=$ Number of donors in each panel.

$+=$ Both tests choose same donor for this ranking.

$0=$ Donor does not occupy the same position of choice by both tests. Best donor placed in column 3 signifies all donors of panel are poor. Donors placed in column 1 and 3 indicate clear distinction between good and poor donor.

Clinical Experience.-The clinical course of the patients whose donors were chosen by these histocompatibility tests is shown in Table IV. From a practical viewpoint it was possible to obtain a result with at least one of the tests in each instance.

TABLB IV.-Correlation of Histocompatibility Tests with Clinical Course

\begin{tabular}{|c|c|c|c|c|c|}
\hline \multirow[b]{2}{*}{ Patient } & \multirow{2}{*}{$\begin{array}{l}\text { Age } \\
\text { and } \\
\text { Sex }\end{array}$} & \multirow[b]{2}{*}{ Donor } & \multicolumn{2}{|c|}{ Match } & \multirow{2}{*}{$\begin{array}{l}\text { Clinical } \\
\text { Result }\end{array}$} \\
\hline & & & N.L.T.* & $\begin{array}{l}\text { Lymphocyte } \\
\text { Stimulationt }\end{array}$ & \\
\hline $\mathbf{Y}$ & $34 \mathrm{M}$ & Brother & Good & Excellent & Alive 10 months. \\
\hline $\mathbf{K n}$ & $39 \mathrm{M}$ & Sister & Excellent & Excellent $\ddagger$ & $\begin{array}{l}\text { Alive } 2 \text { years. Minimal } \\
\text {. }\end{array}$ \\
\hline Sz & $34 \mathrm{M}$ & Brother & Good & - & $\begin{array}{l}\text { rejection crisis } \\
\text { Alive } 3 \text { years. Moderately }\end{array}$ \\
\hline L & $47 \mathrm{M}$ &. & ," & Good & $\begin{array}{l}\text { severe rejection crisis } \\
\text { Alive } 2 \text { years. Severe } \\
\text { rejection crisis }\end{array}$ \\
\hline $\begin{array}{l}\mathrm{V} \\
\mathrm{Q}\end{array}$ & $\begin{array}{l}30 M \\
22 M\end{array}$ & Father & $\begin{array}{l}\text { Unsatisfac- } \\
\text { tory test }\end{array}$ & ", & $\begin{array}{l}\text { Rejected } 3 \text { months } \\
\Rightarrow 8 \%\end{array}$ \\
\hline A & $\begin{array}{ll}7 & F\end{array}$ & $\begin{array}{l}\text { Father } \\
\text { Cadaver }\end{array}$ & Fairly good & Fairly good & Rejected; died $5 \frac{1}{2}$ months \\
\hline G & $16 M$ & Uncle & $\begin{array}{l}\text { Unsatisfac- } \\
\text { tory test }\end{array}$ & Poor & $\begin{array}{l}\text { Died } 2 \text { months of pulmon- } \\
\text { ary infection }\end{array}$ \\
\hline
\end{tabular}

- Normal lymphocyte transfer test. Excellent match $=$ No reaction when patient's cells injected into donor's skin. Good match $=$ Reaction with patient's cells much smaller than with unrelated lymphocytes. Fairly good match $=$
Reaction with patient's cells slightly smaller than with unrelated lymphocytes. Reaction with patient's cells slightly smuller than with unrelated lymphocytes.
Poor match = Reaction with patient's cells same size as with unrelated lymphocytes.

$\dagger$ Mixed lymphocyte culture test. Excellent match $=$ No net stimulation in donor-recipient culture. Good match $=$ Donor-recipient culture stimulation less than one-quarter of that obtained in unrelated-recipient mixtures. Fairly good match $=$ Donor-recipient culture stimulation one-quarter to five-eighths that obtained in unrelated-recipient mixtures. Poor match $=$ Donor-recipient culture stimulation more than five-eighths that obtained in unrelated-recipient mixtures.

₹ Tested in retrospect after the transplant.

\section{Discussion}

A relation has been found between the results of the normal lymphocyte transfer test and those derived from in-vitro lymphocyte stimulation, particularly in selecting the donor of first choice. From first principles, however, one would not necessarily expect the two tests to rank the donors in the same order. The mixed lymphocyte culture test used here involves mutual stimulation by two populations of living cells. The extent to which the host cells contribute to the normal lymphocyte transfer reaction at 48 hours is still a matter of dispute. If in fact the normal lymphocyte transfer reaction is produced in man largely or entirely as the consequence of injected cells recognizing foreign antigens on the cells of the host and reacting against them, as appears to be the case in the guinea-pig (Brent and Medawar, 1964), it would be unlikely that the results of the two tests would correspond closely.

If, on the other hand, the host cells are playing an immunological part which contributes to the cutaneous inflammatory response, as argued by Amos et al. (1965) and Aisenberg (1965), then good correlation between the two tests might be anticipated. Harris et al. (1966) showed a correlation between the mixed live lymphocyte cultures at seven days and the simultaneous normal lymphocyte transfer test which reached significance when induration plus erythema were taken into account, but not if induration alone was considered.

There are at least two major theoretical factors at work in determining the outcome of each transplant in Table IV: the histocompatibility match, and the relation between the individual recipient's sensitivity to immunosuppressive therapy and the dose given. It must be pointed out that Patients $\mathrm{Y}$ and $\mathrm{Kn}$ differed from the majority in that steroids were begun on the second and fourth days respectively instead of at the first sign of rejection. Nevertheless, the association of these two best clinical results with excellent histocompatibility matches is striking. In both these cases there was no net reaction at all between the lymphocytes of donors and recipients in culture. This phenomenon has been recorded by Bain and Lowenstein (1964) in five pairs of siblings. With Patient $\mathrm{Kn}$ this absence of a reaction between cultured lymphocytes corresponded with a completely negative normal lymphocyte transfer reaction in that the recipient's lymphocytes produced no detectable reaction when injected into the donor skin.

In the experiments with volunteers who were skin-grafted a similar absence of lymphocyte stimulation between siblings has been observed on two occasions, and has been shown to correspond to a strikingly prolonged skin-graft-survival time (Russell, 1966). An absence of mutual stimulation by donor and recipient lymphocytes suggests a high degree of histocompatibility. It is therefore of particular interest that the two patients showing this absence of a reaction should be those who had the most favourable postoperative course. In the case of Patient $\mathrm{Y}$ no rejection reaction could be detected in the first 10 months.

The poor results with Patients $\mathrm{V}$ and $\mathrm{Q}$ despite a good histocompatibility match may be explicable by the intolerance of these two patients, together with $A$ and $G$, of doses of azothioprine which have been regarded as normal on a bodyweight basis, and which were readily administered to the other four patients.

\section{Conclusions}

Further experience with the normal lymphocyte transfer test as a routine procedure confirms the earlier conclusion of Gray and Russell (1965) that it is of some value in distinguishing major differences between good and bad donors. Its practical value is increased by the use of adequate controls and by the filtration method of preparing the cells. Its principal limitations remain the difficulty of quantitating the size of the reactions and the failure to detect minor differences. It is likely to be largely superseded by in-vitro tests, possibly a modification of lymphocyte stimulation. The latter test appears at present to possess greater discrimination, and precise objective quantitation is achieved by uptake of radioactive precursors of nucleic acids. It has the additional advantages that it does not sensitize the potential donors, may be repeated any number of times, and does not involve the theoretical hazard of transferring injected material from a sick patient to healthy donors.

The expert technical assistance of Mrs. L. T. Baker and Miss A. Andrade and the help of Dr. B. A. Barnes with analysis of the data are gratefully acknowledged. Three of the normal lymphocyte transfer tests included in Table IV were performed by Mr. J. G. Gray.

\section{REFERENCES}

Aisenberg, A. C. (1965). F. clin. Invest., 44, 555.
Amos, D. B., Nicks, P. J., Peacocke, N., and Sieker, H. O. (1965). Ibid., 44, 219. 
Bach, F., and Hirschhorn, K. (1964). Science, 143, 813.

Bain, B., and Lowenstein, L. (1964). Ibid., 145, 1315.

Bain, B., and Lowenstein, L. (1964), Ibid., 145, 1315. $23,108$.

Brent, L., and Medawar, P. B. (1963). Brit. med. F., 2, 269.

Brent, L., and Medawar, P. B. (1963). Brit.

Bridges, J. M., Nelson, S. D., and McGeown, M. G. (1964). Lancet, 1, 581 .

Chen, P. S. (1958). Proc. Soc. exp. Biol. (N.Y.), 98, 546.

Gray, J. G., and Russell, P. S. (1963). Lancet, 2, 863.

(1965). In Histocompatibility Testing, edited by P. S. Russel and H. J. Winn. Publication No. 1229, Nat. Acad. Sci., Washington, p. 105 Harris, R., Clarke, C. A., Jones, A. L., Sheppard, P. M., Lehane, D.,
McCarthy, M., Lawler, S. D., and Shatwell, H. S. (1966). Brit. med. F., 1, 509.

Hirschhorn, $\mathrm{K}$ (1965). In Histocompatibility Testing, edited by P. S. Russell and H. J. Winn, Publication No. 1229, Nat. Acad. Sci., Washington, p. 177 .

Huggins, C. E. (1964). Ann. Surg., 160, 643.

Johnson, G. J., and Russell, P. S. (1965). Nature (Lond.), 208, 343.

Moorhead, J. F., and Patel, A. R. (1964). Brit. med. 7., 2, 1111

Russell, P. S. (1966). In Histocompatibility Testing 1965 , edited by $\mathbf{H}$ Balner, F. J. Cleton, and J. G. Eernisse, p. 233. Copenhagen.

Streilein, J. W. (1966). Ibid., p. 241.

\title{
Conversion of Cortisone to Cortisol and Prednisone to Prednisolone
}

\author{
J. S. JENKINS,* M.D., M.R.C.P. ; P. A. SAMPSON, $\dagger$ M.B., CH.B.
}

Brit. med.f., 1967, 2, 205-207

Though cortisone has been widely used since Hench et al. (1949) described its value in the treatment of rheumatoid arthritis, there is now considerable evidence that cortisone and other steroids which possess the 11-oxo group instead of a $11 \beta$-hydroxyl are themselves biologically inactive. The direct application of cortisone to the skin is ineffective in the treatment of skin diseases responsive to cortisol (Robinson and Robinson, 1956), and when cortisone is injected locally into inflamed joints its anti-inflammatory action is slight compared with that of cortisol (Hollander et al., 1951). It is the reduction of the 11-oxo to the $11 \beta$-hydroxyl by the enzyme $11 \beta$-hydroxydehydrogenase which renders the 11-oxo steroids cortisone and its synthetic analogue prednisone biologically active when they are administered systemically. In man this reaction appears to take place mainly in the liver (Jenkins, 1966). Several investigators have made clinical comparisons of the therapeutic effects of cortisone and cortisol and of prednisone and prednisolone, but there is less information about the actual amounts of $11 \beta$-hydroxysteroids which are formed from the 11-oxo compounds. Peterson et al. (1957b) described the conversion of cortisone to cortisol in one patient, but the results were complicated by the prior administration of $9 \alpha$-fluoroprednisolone to the patient. Bush and Mahesh (1964) studied the reduction of prednisone, again in only one patient, but no comparison was made with the levels of prednisolone in the plasma after prednisolone itself. The present paper describes the extent to which oral cortisone and prednisone are converted to cortisol and prednisolone in a series of normal subjects, and also in patients suffering from liver disease.

\section{Procedure and Methods}

Nine normal subjects, three patients with Addison's disease, and four patients suffering from disease of the liver, were given single oral doses of cortisone or prednisone as crushed tablets in amounts ranging from 25 to $200 \mathrm{mg}$. On subsequent occasions a similar dose of cortisol or prednisolone was given to the same subjects. At hourly intervals after the administration of cortisone and cortisol the levels of cortisol in the plasma were compared. Similarly, the levels of prednisolone in the plasma were measured after giving prednisone and prednisolone. Cortisone was administered mostly as the acetate, since this is the usual form available for therapeutic use, but in some instances the free alcohol was also given for comparison. Cortisol, prednisone, and prednisolone were given as the free alcohol.

\footnotetext{
* Consultant Physician, St. George's Hospital, London S.W.1.
}

t Research Assistant, St. George's Hospital, London S.W.1.
After their oral administration the absorption of each of the pair of steroids was compared by measuring the total 24-hour excretion of 17-oxogenic steroids in the urine.

Cortisol in Plasma.-A fluorimetric technique (Rudd et al., 1963) was used, by means of which cortisol can be measured without interference from cortisone or other metabolites. In some cases plasma cortisol, tetrahydrocortisol, cortisone, and tetrahydrocortisone were estimated specifically by a paper chromatographic technique similar to that described for the estimation of plasma prednisolone, the appropriate standard steroids being used.

Prednisolone in Plasma.-Five millilitres of heparinized plasma was extracted with $25 \mathrm{ml}$. of methylene chloride, and the extract was washed first with $2 \mathrm{ml}$. of 0.1 normal sodium hydroxide, followed by 0.1 normal acetic acid and finally with water. The extract was evaporated to dryness, redissolved in a small volume of a mixture of methanol and methylene chloride, and applied to Whatman No. 1 chromatography paper. Chromatograms were run for four hours in the benzenemethanol-water (100:50:50) system of Bush (1952). The prednisolone area was located by ultraviolet light and eluted with methanol from the chromatogram. The eluate was taken to dryness, redissolved in $0.5 \mathrm{ml}$. of methylene chloride, and the steroid estimated, the phenylhydrazinesulphuric acid reagent of Porter and Silber (1950) being used according to the technique of Peterson et al. (1957a). Standard amounts of prednisolone were taken through the whole procedure.

17-Oxogenic steroids in the urine were estimated by the method of Appleby et al. (1955).

\section{Conversion of Cortisone to Cortisol}

The mean levels of cortisol in the plasma after the oral administration of 100 or $200 \mathrm{mg}$. of cortisone and cortisol to normal subjects and $25 \mathrm{mg}$. of these steroids to patients with Addison's disease are shown in Fig. 1 . No significant difference was seen in the levels obtained with cortisone acetate and the free alcohol when both were given to some of the subjects on different occasions, and they have therefore not been recorded separately. The peak level after cortisone was normally at two hours, but in all cases was lower than the peak after cortisol. This difference became disproportionately greater as the dosage increased up to $200 \mathrm{mg}$., when plasma cortisol after cortisone was only a third to half the level after cortisol. The various unconjugated steroids present in the plasma of a normal subject two hours after $200 \mathrm{mg}$. of cortisone and cortisol were examined chromatographically, and are shown in Table I. The cortisol 